\title{
Rapid Increase in Circulation of the SARS-CoV-2 B.1.617.2 (Delta) Variant - Mesa County, Colorado, April-June 2021
}

\begin{abstract}
Rachel Herlihy, $\mathrm{MD}^{1}$; Wendy Bamberg, $\mathrm{MD}^{1}$; Alexis Burakoff, $\mathrm{MD}^{1}$; Nisha Alden, $\mathrm{MPH}^{1}$; Rachel Severson, $\mathrm{MS}^{1}$; Eric Bush, DVM ${ }^{1}$; Breanna Kawasaki, $\mathrm{MPH}^{1}$; Brynn Berger, $\mathrm{MPH}^{1}$; Elizabeth Austin, $\mathrm{MPH}^{1}$; Meghan Shea, MPH ${ }^{1}$; Eduardo Gabrieloff, MFA ${ }^{1}$; Shannon Matzinger, PhD ${ }^{1}$; April Burdorf, $\mathrm{MPH}^{1}$; Janell Nichols ${ }^{1}$; Kim Goode ${ }^{1}$; Alana Cilwick, $\mathrm{MPH}^{1}$; Chelsea Stacy, $\mathrm{MPH}^{2}$; Erin Staples, MD, PhD 3 ; Ginger Stringer, PhD ${ }^{1}$
\end{abstract}

On August 6, 2021, this report was posted as an MMWR Early Release on the MMWR website (https://www.cdc.gov/mmwr).

On May 5, 2021, the Colorado Department of Public Health and Environment (CDPHE) identified the first five COVID-19 cases caused by the SARS-CoV-2 B.1.617.2 (Delta) variant in Mesa County in western Colorado (population 154,933, <3\% of the state population). All five initial cases were associated with school settings. Through early June, Mesa County experienced a marked increase in the proportion of Delta variant cases identified through sequencing: the 7-day proportion of sequenced specimens identified as B.1.617.2 in Mesa County more than doubled, from $43 \%$ for the week ending May 1 to $88 \%$ for the week ending June 5 . As of June 6, more than one half (51\%) of sequenced B.1.617.2 specimens in Colorado were from Mesa County. CDPHE assessed data from surveillance, vaccination, laboratory, and hospital sources to describe the preliminary epidemiology of the Delta variant and calculate crude vaccine effectiveness (VE). Vaccination coverage in early May in Mesa County was lower (36\% of eligible residents fully vaccinated) than that in the rest of the state (44\%). Compared with that in all other Colorado counties, incidence, intensive care unit (ICU) admissions, and COVID-19 case fatality ratios were significantly higher in Mesa County during the analysis period, April 27-June 6, 2021. In addition, during the same time period, the proportion of COVID-19 cases in persons who were fully vaccinated (vaccine breakthrough cases) was significantly higher in Mesa County compared with that in all other Colorado counties. Estimated crude VE against reported symptomatic infection for a 2 -week period ending June 5 was $78 \%$ (95\% confidence interval $[\mathrm{CI}]=71 \%-84 \%)$ for Mesa County and $89 \%$ (95\% CI $=88 \%-91 \%)$ for other Colorado counties. Vaccination is a critical strategy for preventing infection, serious illness, and death from COVID-19. Enhanced mitigation strategies, including masking in indoor settings irrespective of vaccination status, should be considered in areas with substantial or high case rates.

Whole genome sequencing is performed in the CDPHE laboratory on specimens submitted as part of sentinel surveillance (38 sites across Colorado, including one acute care hospital in Mesa County), as well as for cluster and outbreak response and on suspected variants (reverse transcription-polymerase chain reaction [RT-PCR]-positive specimens with S-gene target failure associated with the B.1.1.7 lineage) (1). The
Colorado Electronic Disease Reporting System (CEDRS), a surveillance system managed by CDPHE, was used to identify reported confirmed or probable cases of COVID-19 occurring from April 27, the date of illness onset for the first Delta variant case in Mesa County, to June 6, when sequencing identified B.1.617.2 as the dominant variant in Colorado (2). The Colorado Immunization Information System (CIIS) was used to verify COVID-19 vaccination status; vaccine breakthrough infections were identified using personally identifying information to match cases in CEDRS to CIIS entries* (3). Crude VE against reported symptomatic infection was estimated and compared among Mesa County and all other Colorado counties using a screening method outlined by the World Health Organization ${ }^{\dagger}$ as a rapid tool to assess whether a vaccine is performing as expected (4). To better determine settings where the Delta variant was spreading, outbreak data during April 22-June 26 were obtained from the CDPHE outbreak database, which contains information on all reported COVID-19 outbreaks in Colorado and outbreak line lists. $\$$ Residential care facility vaccination data were obtained from

\footnotetext{
* SARS-CoV-2 RNA or antigen in a respiratory specimen collected from a person $\geq 14$ days after they have completed all recommended doses of the primary series for a Food and Drug Administration-authorized COVID-19 vaccine.

$\dagger$ Crude VE was estimated as (1-[\{PCV/(1-PCV)\}/\{PPV/(1-PPV)\}]) following World Health Organization interim guidance on conducting VE evaluations in the setting of new SARS-CoV-2 variants where PCV is the observed percentage of cases in persons who are vaccinated and PPV is the percentage of a comparable group in the population who are vaccinated. The PPV used in the calculations for Mesa County and other Colorado counties was from May 7, 2021, approximately 2 weeks before the anticipated onset for cases included in the PCV estimate. PPV included only vaccine-eligible persons and PCV was limited to symptomatic persons who were vaccine-eligible.

$\S$ An outbreak in a residential care facility (skilled nursing facility, assisted living residence, intermediate care facility, or group home) is defined as the occurrence of two or more confirmed cases of COVID-19 among residents and staff members in a facility within 14 days, or one confirmed case and two or more probable cases of COVID-19 among residents and staff members in a facility within 14 days. Until May 31, 2021, the definition of a school outbreak was defined as two or more confirmed COVID-19 cases among students, teachers, and staff members from separate households within 14 days in a single classroom, cohort, or activity or other close contact in the school setting; or one confirmed case and two or more probable cases of COVID-19 among students, teachers, and staff members from separate households within 14 days in a single classroom, cohort, or activity or other close contact in the school setting. Starting June 1, the definition changed from two or more to five or more cases of COVID-19, of which at least one patient has had a positive molecular amplification test or antigen test, among students, teachers, and staff members from separate households within 14 days in a single classroom, cohort, or activity or other close contact in the school setting.
} 
EMResource, a capacity planning tool used by CDPHE for facility-level reporting of aggregate COVID-19 vaccinations. Incidence of SARS-CoV-2 infection and proportions of outcomes and vaccination rates among patients living in Mesa County and all other Colorado counties were compared and p-values were calculated using chi-square or Fisher's exact tests. This activity was reviewed by CDC and was conducted consistent with applicable federal law and CDC policy.

During April 27-June 6, a total of 1,945 COVID-19 cases were reported in Mesa County through CEDRS (incidence $=1,255$ per 100,000). Compared with that in all other Colorado counties, incidence, overall ICU admissions, and overall case fatality ratios were significantly higher in Mesa County (Table). In addition, the proportion of breakthrough cases was significantly higher in Mesa County than in all other Colorado counties. In Mesa County, the proportion of persons aged $\geq 65$ years with COVID-19 who were fully vaccinated $(27.5 \%)$ was significantly higher than that in all other Colorado counties (17.4\%). The crude VE against reported symptomatic infection for a 2-week period ending June 5 was $78 \%(95 \% \mathrm{CI}=71 \%-84 \%)$ for Mesa County and $89 \%(95 \% \mathrm{CI}=88 \%-91 \%)$ for all other Colorado counties. ${ }^{* *}$

Among 18,475 sequenced specimen results reported in Colorado through June 6, a total of 783 infections with the Delta variant were identified; more than one half $(400 ; 51.1 \%)$ of these occurred among Mesa County residents, even though the county accounts for $<3 \%$ of the state's population. Symptomatic illness was reported in $304(76.0 \%)$ of the 400 Delta variant infections in Mesa County residents and 251 (65.5\%) of 383 Delta variant infections in other counties. The 7-day percentage of sequenced sentinel specimens identified as SARS-CoV-2 B.1.617.2 in Mesa County increased from $43 \%$ for the week ending May 1 to $88 \%$ for the week ending June 5 (Figure). During the 5-week period, 67\% (51 of 76) of sentinel surveillance specimens in Mesa County were identified as B.1.617.2 compared with 15\% (248 of 1,637) of specimens from all other Colorado counties sequenced over the same time frame.

During April 22-June 26, a total of 37 COVID-19 outbreaks were reported in Mesa County; 13 (35\%) in residential care facilities, $11(30 \%)$ in schools, two $(5 \%)$ in correctional facilities, and $11(30 \%)$ in other settings. Twelve outbreaks, including seven in residential care facilities, had at least one Delta variant case. Average vaccination coverage in these seven residential facilities was $87 \%$ among residents (range $=50 \%-97 \%$ ) and $50 \%$ among staff members (range $=6 \%-69 \%$ ); attack rates

\footnotetext{
45 C.F.R. part 46, 21 C.F.R. part 56; 42 U.S.C. Sect. 241(d); 5 U.S.C. Sect. 552a; 44 U.S.C. Sect. 3501 et seq.

** For Mesa County, PPV was $36.2 \%$ and PCV was $11.0 \%$. For other Colorado counties, PPV was $44.2 \%$ and PCV was $7.9 \%$.
}

\section{Summary \\ What is already known about this topic?}

The highly transmissible B.1.617.2 (Delta) variant of SARS-CoV-2 has become the predominant circulating U.S. strain.

What is added by this report?

During April-June 2021, COVID-19 cases caused by the Delta variant increased rapidly in Mesa County, Colorado. Compared with that in other Colorado counties, incidence, intensive care unit admissions, COVID-19 case fatality ratios, and the proportion of cases in fully vaccinated persons were significantly higher in Mesa County. Crude vaccine effectiveness against symptomatic infection was estimated to be $78 \%$ for Mesa County and $89 \%$ for other Colorado counties.

What are the implications for public health practice?

Vaccination is critical for preventing infection, serious illness, and death associated with SARS-CoV-2 infection (including the Delta variant). Multicomponent prevention strategies, such as masking in indoor settings irrespective of vaccination status as well as optimal surveillance testing and infection prevention and control, should be considered in areas of high incidence.

among residents ranged from $0 \%$ to $54.6 \%$ (median $=1.2 \%)$ and among staff members from $2.2 \%$ to $25.5 \%$ (median $=10.0 \%$ ). Five of these seven outbreaks involved at least one case in a fully vaccinated resident or staff member. ${ }^{\dagger \dagger}$

\section{Discussion}

The Delta variant is highly transmissible; within 5 weeks of first identification, the Delta variant became the dominant SARS-CoV-2 variant in Mesa County, Colorado and is also now the predominant variant in the United States (5). Higher ICU admissions and case fatality ratios in Mesa County compared with those in the rest of the state are consistent with previous reports that infections with the Delta variant might result in more severe outcomes $(6,7)$. The slightly lower crude VE estimate against symptomatic infection in Mesa County may lend support to previous findings that COVID-19 vaccines provide modestly lower protection against symptomatic infection with the Delta variant (8). Alternatively, because the Delta variant was circulating at higher levels in Mesa County than in other Colorado counties, the lower VE in Mesa County might reflect the much higher exposure to circulating virus among vaccinated persons.

The findings in this report are subject to at least four limitations. First, lack of genetic sequencing for all SARS-CoV-2 isolates likely affected estimated rates and proportions; the number of outbreaks involving the Delta variant might be

\footnotetext{
${ }_{\dagger}$ A fully vaccinated person is one who has completed all recommended doses of an FDA-authorized COVID-19 vaccine, including Pfizer-BioNTech, Moderna, and Janssen (Johnson \& Johnson) $\geq 14$ days before a positive SARS-CoV-2 test result.
} 
TABLE. Age-specific incidence, clinical outcomes, and vaccination status among COVID-19 cases in Mesa and other counties - Colorado, April 27-June 6, 2021

\begin{tabular}{|c|c|c|c|}
\hline Characteristic & Mesa County & Other Colorado counties & p-value ${ }^{\dagger}$ \\
\hline Total COVID-19 cases, no. & 1,945 & 35,494 & - \\
\hline \multicolumn{4}{|l|}{ Age group, yrs } \\
\hline $0-17$ & 477 & 7,603 & - \\
\hline $18-64$ & 1,246 & 25,466 & - \\
\hline$\geq 65$ & 222 & 2,425 & - \\
\hline Overall incidence* & 1,255 & 633 & $<0.001$ \\
\hline \multicolumn{4}{|l|}{ Age group, yrs } \\
\hline $0-17$ & 1,408 & 620 & $<0.001$ \\
\hline $18-64$ & 1,377 & 714 & $<0.001$ \\
\hline$\geq 65$ & 726 & 297 & $<0.001$ \\
\hline Hospital admission, no./No. (\%) & $142 / 1,945(7.3)$ & $2,448 / 35,494(6.9)$ & 0.49 \\
\hline \multicolumn{4}{|l|}{ Age group, yrs } \\
\hline $0-17$ & $3 / 477(0.6)$ & $97 / 7,603(1.3)$ & 0.22 \\
\hline $18-64$ & $69 / 1,246(5.5)$ & $1,554 / 25,466(6.1)$ & 0.42 \\
\hline$\geq 65$ & $70 / 222(31.5)$ & $797 / 2,425$ (32.9) & 0.69 \\
\hline ICU admission among hospitalized patients, no./No. (\%) & $49 / 142(34.5)$ & $583 / 2,448(23.8)$ & 0.004 \\
\hline \multicolumn{4}{|l|}{ Age group, yrs } \\
\hline $0-17$ & $1 / 3(33.3)$ & $17 / 97(17.5)$ & 0.45 \\
\hline $18-64$ & $25 / 69(36.2)$ & $356 / 1,554(22.9)$ & 0.01 \\
\hline$\geq 65$ & $23 / 70(32.9)$ & 210/797 (26.4) & 0.24 \\
\hline Overall CFR, no./No. (\%) & $29 / 1,945(1.5)$ & $299 / 35,494(0.8)$ & 0.003 \\
\hline \multicolumn{4}{|l|}{ Age group, yrs } \\
\hline $0-17$ & $1 / 477(0.2)$ & $2 / 7,603(0.03)$ & 0.16 \\
\hline $18-64$ & $7 / 1,246(0.6)$ & $101 / 25,466(0.4)$ & 0.37 \\
\hline$\geq 65$ & $21 / 222(9.5)$ & $196 / 2,425(8.1)$ & 0.47 \\
\hline CFR, hospitalized patients, no./No. (\%) & $22 / 142(15.5)$ & $198 / 2,448(8.1)$ & 0.002 \\
\hline \multicolumn{4}{|l|}{ Age group, yrs } \\
\hline $0-17$ & $1 / 3(33.3)$ & $1 / 97(1.0)$ & 0.06 \\
\hline $18-64$ & $5 / 69(7.2)$ & $55 / 1,554(3.5)$ & 0.11 \\
\hline$\geq 65$ & $16 / 70(22.9)$ & $142 / 797(17.8)$ & 0.29 \\
\hline Fully vaccinated ${ }^{\S, \pi}$, no./No. (\%) & $136 / 1,945(7.0)$ & $1,715 / 35,397(4.8)$ & $<0.001$ \\
\hline \multicolumn{4}{|l|}{ Age group, yrs } \\
\hline $0-17$ & $2 / 477(0.4)$ & $10 / 7,591(0.1)$ & 0.16 \\
\hline $18-64$ & $73 / 1,246(5.9)$ & $1,283 / 25,381(5.1)$ & 0.21 \\
\hline$\geq 65$ & $61 / 222(27.5)$ & $422 / 2,425(17.4)$ & $<0.001$ \\
\hline
\end{tabular}

Abbreviations: $\mathrm{CFR}=$ case fatality ratio; ICU = intensive care unit.

* Cases per 100,000 population.

${ }^{\dagger}$ Calculated using chi-square test or Fisher's exact test.

$\S$ A fully vaccinated person is one who has completed all recommended doses of a Food and Drug Administration-authorized COVID-19 vaccine, including Pfizer-BioNTech, Moderna, and Janssen (Johnson \& Johnson) $\geq 14$ days before a positive SARS-Co-V-2 test result.

" Vaccination status was missing for 97 persons.

underreported for this reason. Second, sentinel surveillance might not provide a fully representative sample of sequence types in Colorado because the specimens originate from hospitals and likely include more specimens from inpatients and emergency department patients compared with specimens from other testing sites. Third, the screening method provides rapid crude VE estimates that do not control for possible effects of confounding or clustering. Some of the differences between VE and severity of illness in Mesa County and that in other counties might be due to differences in the age distribution of patients and the inclusion of cases associated with outbreaks in congregate settings. However, CDPHE estimates that fewer than $10 \%$ of cases during the time period occurred in congregate settings. Finally, differences in vaccination coverage in some of these populations might be an additional confounding factor when estimating crude VE at the county and state levels. VE studies with more rigorous methods and the power to estimate protection against severe outcomes are needed to better understand the potential impact of the Delta variant.

Vaccination is a critical strategy for preventing infection, serious illness, and death associated with SARS-CoV-2 (including the Delta variant). Additional targeted prevention strategies (e.g., masking in indoor settings irrespective of vaccination status) and adherence to prevention strategies (e.g., surveillance testing and infection prevention and control procedures) are prudent in areas with high circulation of the Delta variant and in higher risk settings, such as residential care facilities. 
FIGURE. Number of COVID-19 cases and proportion of B.1.617.2 (Delta) variant infections in Mesa and other counties - Colorado, April 27-June 6, 2021

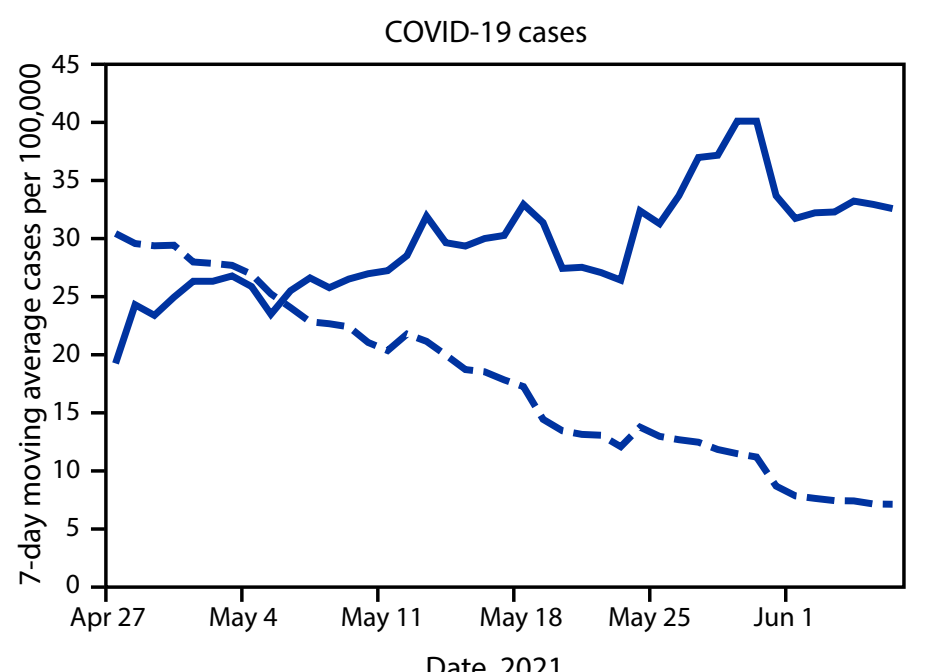

Date, 2021

Corresponding author: Rachel Herlihy, rachel.herlihy@state.co.us.

${ }^{1}$ Colorado Department of Public Health and Environment; ${ }^{2}$ Mesa County Public Health Department, Grand Junction, Colorado; ${ }^{3}$ CDC COVID-19 Response Team.

All authors have completed and submitted the International Committee of Medical Journal Editors form for disclosure of potential conflicts of interest. Wendy Bamberg reports receipt of payment for Grand Rounds presentation on COVID-19 in April 2020 and membership on the Medical Advisory Board for First Descents. No other potential conflicts of interest were disclosed.

\section{References:}

1. Martin Webb L, Matzinger S, Grano C, et al. Identification of and surveillance for the SARS-CoV-2 variants B.1.427 and B.1.429Colorado, January-March 2021. MMWR Morb Mortal Wkly Rep 2021;70:717-8. PMID:33988184 https://doi.org/10.15585/mmwr. $\mathrm{mm} 7019 \mathrm{e} 2$

2. Turner K, Davidson SL, Collins J, et al. Update to the standardized surveillance case definition and national notification for 2019 novel coronavirus disease (COVID-19). Atlanta, GA: Council of State and Territorial Epidemiologists; 2020. https://cdn.ymaws.com/www.cste.org/resource/resmgr/ps/ positionstatement2020/Interim-20-ID-02_COVID-19.pdf

3. CDC. COVID-19 vaccine breakthrough case investigation and reporting. Atlanta, GA: US Department of Health and Human Services, CDC: 2021. Accessed July 1, 2021. https://www.cdc.gov/vaccines/covid-19/ health-departments/breakthrough-cases.html

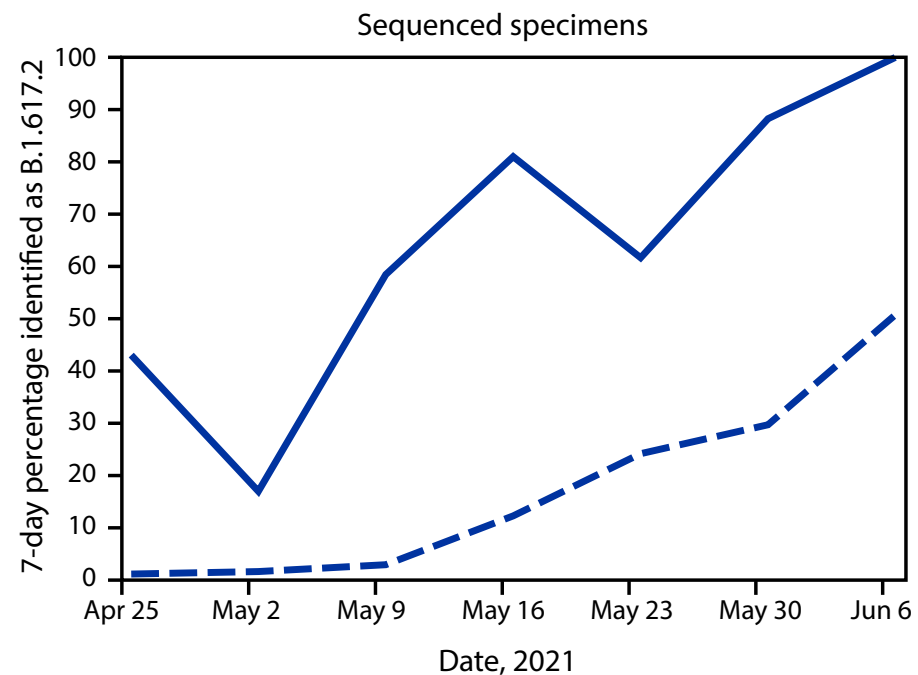

Date, 2021

4. World Health Organization. Guidance on conducting vaccine effectiveness evaluations in the setting of new SARS-CoV-2 variants: interim guidance, 22 July 2021: addendum to evaluation of COVID-19 vaccine effectiveness, 2021. Geneva, Switzerland: World Health Organization; 2021. https://www.who.int/publications/i/item/ WHO-2019-nCoV-vaccine_effectiveness-variants-2021.1

5. Public Health England. SARS-CoV-2 variants of concern and variants under investigation in England: technical briefing 17. London, United Kingdom: Public Health England; 2021. https://assets.publishing.service. gov.uk/government/uploads/system/uploads/attachment_data/ file/1001354/Variants_of_Concern_VOC_Technical_Briefing_17.pdf

6. Sheikh A, McMenamin J, Taylor B, Robertson C; Public Health Scotland and the EAVE II Collaborators. SARS-CoV-2 Delta VOC in Scotland: demographics, risk of hospital admission, and vaccine effectiveness. Lancet 2021;397:2461-2. PMID:34139198 https://doi.org/10.1016/ S0140-6736(21)01358-1

7. Public Health England. SARS-CoV-2 variants of concern and variants under investigation in England: technical briefing 15. London, United Kingdom: Public Health England; 2021. https://assets.publishing.service. gov.uk/government/uploads/system/uploads/attachment_data/ file/993879/Variants_of_Concern_VOC_Technical_Briefing_15.pdf

8. Lopez Bernal J, Andrews N, Gower C, et al. Effectiveness of Covid-19 vaccines against the B.1.617.2 (Delta) variant. N Engl J Med 2021. Epub July 21, 2021. PMID:34289274 https://doi.org/10.1056/ NEJMoa2108891 